\title{
GENERIC DEFORMATIONS OF VARIETIES
}

BY

YIEH-HEI WAN ${ }^{1}$

\begin{abstract}
Typical families of varieties, which are defined by families of smooth maps, are studied through the method from the singularity theory of differentiable maps. It is proved that generic families of varieties (of certain types) are stable in an appropriate sense.
\end{abstract}

1. Introduction. Let $U$ be an open set in $R^{p}$ and $M$ be an open set in $R^{s}$ with origin 0 in $M$. Now, consider a smooth family $\left\{f_{u}\right\}$ of smooth mappings from $M$ into $R^{t}$ with parameters $u$ lying in $U$. Equivalently, this family $\left\{f_{u}\right\}$ can be described as a single smooth map $f: U \times M \rightarrow R^{t}$, where $f(u, x)=f_{u}(x)$. For each $u$ in $U$, we have a "variety" $f_{u}^{-1}(0)$ in $M$ defined by $f_{u}$. The problems which we shall investigate are the following.

(A) What are the typical local deformations $\left\{f_{u}^{-1}(0)\right\}$ of varieties for smooth maps $f: U \times M \rightarrow R^{t}$ as $u$ varies within $U$ ?

(B) What are the typical local deformations $\left\{f_{u}^{-1}(0)\right\}$ of varieties near $U \times\{0\}$ for smooth maps $f: U \times M \rightarrow R^{t}$ with $f(U, 0)=0$ as $u$ varies in $U$ ?

Notice that we only study maps $f_{u}: M \rightarrow R^{t}$ with $f_{u}(0)=0$ in problem (B). Thus, problem (B) can be regarded as a relative version of problem (A). A clear distinction between these two problems is that, typically, the set $f^{-1}(0)$ is a submanifold in problem (A) but it is not a submanifold in problem (B).

Besides being very attractive mathematical questions, problems (A) and (B) arise naturally in a number of occasions such as in the studies of differential equations and economics. Suppose that a family of ordinary differential equations $d x / d t=$ $f_{u}(x)=f(u, x)$ on $M$ is given, where $f: U \times M \rightarrow R^{s}$ depends on $u, x$ smoothly. Sometimes this family $f_{u}(x)$ has the additional property, namely $f_{u}(0)=0$ for all $u$. For any $u, x$ in $M$ is a stationary solution of the equation $d x / d t=f_{u}(x)$ if and only if $x$ belongs to the variety $f_{u}^{-1}(0)$. Therefore, the bifurcation problems about stationary solutions of ordinary differential equations $d x / d t=f_{u}(x)$ become the special cases of problems (A) and (B) where $s$ equals $t$. Next, consider an economic model of $s+1$ goods with an aggregate excess demand function $f_{u}: M \rightarrow R^{s}$ depending on a shift parameter $u$ in $U$. Here $M$ is the set of all admissible prices of these $s+1$ goods. From the works of Sonnenschein, Debreu [1] and others, it seems reasonable to take $\left\{f_{u}\right\}$ as a smooth family of smooth mappings. A point $p$ in $M$ is an equilibrium price if and only if $f_{u}(p)=0$. Consequently, the problem of

Received by the editors November 27, 1978.

AMS (MOS) subject classifications (1970). Primary 58C25.

${ }^{1}$ Research partially supported by the National Science Foundation under grant MCS 7701087.

(C) 1980 American Mathematical Society 0002-9947/80/0000-0206/\$04.25 
comparative statics for these economic models becomes the special case of problem (A) where $s$ equals $t$.

Motivated by the works of Whitney, Thom, Mather and others on singularities of differentiable mappings [4], [5], we expect that if the dimension $p$ of the parameter space $U$ is small then typical local (relative) deformations of varieties exhibit stability and universality properties in a suitable smooth setting. Furthermore, one can find a finite list for these typical local (relative) deformations of varieties. Roughly speaking, that is the content of Theorems 1 and 3 which we plan to prove in this paper. When the dimension $p$ of the parameter space $U$ becomes large, we expect that typical local deformations of varieties only exhibit stability properties in appropriate topological settings (cf. Examples 2, 3 in §5). Our Theorems 2 and 4 show that it is indeed the case. Precise statements of all these results (i.e. Theorems 1 to 4 ) are given in $\$ 2$.

In order to prove these generic results on deformations of varieties and relative deformations of varieties, two parametrized versions of Thom's transversality theorem of jet bundles are introduced in $\$ \S 4,5$ as Propositions 3, 4 respectively. The problem now is to find appropriate stratification in various "jet bundles" so that each $f: U \times M \rightarrow R^{t}$, whose jet extension of $f$ is transversal to this stratification, has the desired stability or universality property locally. In $\S 4$, problem (A) concerning typical local deformations of varieties is considered and its answers to Theorems 1 and 2 are justified. When the dimension $p$ of the parameter space $U$ is small, the notion of contact equivalence, which has been introduced by Mather in [4], is used to define a stratification of a jet bundle. To show this stratification is the right one, one needs a result of Martinet [3], which says that $V$-universal deformations are the same as $V$-transversal deformations. When $p$ becomes large, the desired stratification can be built from the stratifications defined in Mather [5] or Looijenga [2]. These stratifications in [5], [2] are constructed for the purpose of proving the genericity about topologically stable mappings. In the final section, problem (B) concerning typical local relative deformations of varieties is studied and its answers to Theorems 3 and 4 are proved. The demonstration of Theorem 3 requires a relative version of a result on $V$-universal deformations in Martinet [3]. We state and justify this result on relative $V$-universal deformations in $\S 3$. The stratifications needed in the proofs of Theorems 3 and 4 are more complicated than those in Theorems 1 and 2. However, the ideas about their constructions are similar. Examples which show that Theorems 1 and 3 may fail when $p$ becomes large are given at the end of this paper.

2. Statements of results. Throughout this section, we fix an open set $U$ in $R^{p}$ and an open set $M$ in $R^{s}$ with $0 \in M$. The space $C^{\infty}\left(U \times M, R^{t}\right)$ of smooth maps $f$ : $U \times M \rightarrow R^{t}$ is endowed with the smooth Whitney topology. For each $f$ in $C^{\infty}\left(U \times M, R^{t}\right)$, the bifurcation map $\pi_{f}: f^{-1}(0) \rightarrow U$ is defined by $\pi_{f}(u, x)=x$.

In order to state Theorems 1,3 we need the notion of $V$-universal deformations and relative $V$-universal deformations of a map germ. Denote by $f_{0}:\left(R^{s}, 0\right) \rightarrow$ $\left(R^{t}, 0\right)$ a smooth map germ with source $0 \in R^{s}$ and target $0 \in R^{t}$. A smooth map 
germ $f:\left(R^{p} \times R^{s}, 0\right) \rightarrow\left(R^{t}, 0\right)$ with $f_{0}(x)=f(0, x)\left(f_{0}(x)=f(0, x)\right.$ and $f(u, 0)=$ 0 ) for $x$ near $0 \in R^{s}$ is called a deformation (relative deformation) on $p$ parameters of the map germs $f_{0}$. Given a smooth map germ $h:\left(R^{q}, 0\right) \rightarrow\left(R^{p}, 0\right), h^{*} f$ : $\left(R^{q} \times R^{s}, 0\right) \rightarrow\left(R^{t}, 0\right)$, defined by $h^{*} f(u, x)=f(h(u), x)$ is called the induced deformation of $f$ via $h$. Denote by $x=\left(x_{1}, \ldots, x_{m}\right)\left(y=\left(y_{1}, \ldots, y_{n}\right)\right)$ the canonical coordinate functions on $R^{m}\left(R^{n}\right)$. Let $\mathcal{E}_{x}\left(\mathcal{E}_{y}\right)$ be the local ring of real-valued smooth functions near 0 in $R^{m}\left(R^{n}\right)$, and $m_{x}\left(m_{y}\right)$ be the ideal generated by $x_{1}, \ldots, x_{m}\left(y_{1}, \ldots, y_{n}\right)$ in $\mathcal{E}_{x}\left(\mathcal{E}_{y}\right)$. For a smooth map germ $\varphi:\left(R^{m}, 0\right) \rightarrow\left(R^{n}, 0\right)$, write $\varphi^{*} m_{y}$ for the ideal generated by $\varphi_{1}, \ldots, \varphi_{n}$ in $\varepsilon_{x}$, where $\varphi=\left(\varphi_{1}, \ldots, \varphi_{n}\right)$.

Definition 1. Let $f, g:\left(R^{p} \times R^{s}, 0\right) \rightarrow\left(R^{t}, 0\right)$ be two (relative) deformations of $f_{0} . f$ is said to be (relative) $V$-equivalent to $g$ if there exists a smooth diffeomorphism germ $\Phi:\left(R^{p} \times R^{s}, 0\right) \rightarrow\left(R^{p} \times R^{s}, 0\right)$ in the form $\Phi(u, x)=(k(u), \varnothing(u, x))$ with $(\varnothing(0, x)=x, \varnothing(u, 0)=0) \varnothing(0, x)=x$ such that $\Phi^{*}\left(g^{*} m_{y}\right)=f^{*} m_{y}$. When $k$ can be chosen to be the identity map, $f$ is said to be (relative) $V$-isomorphic to $g$. A deformation $f$ of $f_{0}$ is said to be (relative) $V$-universal iff for any (relative) deformation $f^{*}$ of $f_{0}, f^{*}$ is (relative) $V$-isomorphic to an induced deformation $h^{*} f^{*}$ via some map germ $h:\left(R^{q}, 0\right) \rightarrow\left(R^{p}, 0\right)$.

Now, we state our first two results.

TheOREM 1. Suppose that $(p+s-t, p)$ is a nice dimension as defined by Mather in $[4, \mathrm{VI}]$ when $p+s-t \geqslant 1$. Then, there exists an open and dense subset $\theta$ in $C^{\infty}\left(U \times M, R^{t}\right)$ such that for any $f \in \mathcal{O}$, one gets

(a) $f^{-1}(0) \subset U \times M$ is a smooth manifold of dimension $p+s-t$ if $f^{-1}(0) \neq \varnothing$,

(b) to each $(u, x) \in f^{-1}(0)$, the germ of $f \circ(\psi \times \varphi)^{-1}$ at $(0,0)$ is a $V$-universal deformation on $p$ parameters, where $\psi \times \varphi$ can be any product chart on $U \times M$ with $(\psi \times \varphi)(u, x)=(0,0)$.

THEOREM 2. There exists an open and dense subset $\theta^{*}$ such that for any $f \in \theta^{*}$, it has the following two properties.

(a) $f^{-1}(0) \subset U \times M$ is a smooth manifold of dimension $p+s-t$ if $f^{-1}(0) \neq \varnothing$.

(b) The bifurcation map $\pi_{f}: f^{-1}(0) \rightarrow U$ defines a topologically stable germ at any $(u, x)$ in $f^{-1}(0)$.

Set $R=\{(m, n) \mid m, n$ are positive integers, $n<7$ or $n \geqslant 7(m-1) / 6\} \cup\{(8,7)$, $(8,8),(14,15),(20,22),(26,29)\} \backslash\{(8,6)\}$. Recall that a pair $(m, n)$ of positive integers is called a nice dimension iff $(m, n)$ belongs to $R$. Given a deformation $f$ : $\left(R^{p} \times R^{s}, 0\right) \rightarrow\left(R^{t}, 0\right)$ with $0 \in R^{t}$ as a regular value, $f^{-1}(0)$ is a smooth manifold near 0. In [3], Martinet shows that $f$ is a $V$-universal deformation iff the map germ $\pi_{f}: f^{-1}(0) \rightarrow U$ at 0 is smoothly stable (cf. Remark 1 ). Thus, universality and stability properties of deformations are the same concept in smooth settings. For a fixed $p$, the $V$-equivalence classes of $V$-universal deformations on $p$ parameters are finite in number (cf. $\$ 3$ and also [5]). From Example 2 in \$5, one obtains that some typical deformations may fail to show stability or universality properties in a smooth setting when $(p+s-t, p)$ is not a nice dimension. However, Theorem 2 tells us that typical deformations exhibit topological stability without any assumptions on the dimensions $p, s$ or $t$. The notion of topologically stable germs can be 
defined as germs of topologically stable maps (cf. [5]).

It remains to formulate relative versions of Theorems 1 and 2 . Denote by $C^{\infty}\left(U \times M, U \times\{0\} ; R^{t},\{0\}\right)$ the subspace of $C^{\infty}\left(U \times M, R^{t}\right)$, which consists of those smooth maps $f: U \times M \rightarrow R^{t}$ with $f(U \times\{0\})=\{0\}$. The appropriate notion of stability in relative cases is given as follows.

Definition 2. Let $f \in C^{\infty}\left(U \times M, U \times\{0\} ; R^{t},\{0\}\right)$ and $(\bar{u}, 0) \in f^{-1}(0)$. The bifurcation map $\pi_{f}: f^{-1}(0) \rightarrow U$ is said to be smoothly (topologically) stable at $(\bar{u}, 0)$ under small perturbations on $f$, if given any neighborhood $N$ of $\bar{u}$ in $U$, for $g$ in $C^{\infty}\left(U \times M, U \times\{0\} ; R^{t},\{0\}\right)$ sufficiently close to $f$, there exist diffeomorphism (homeomorphism) germs $\alpha:(U \times M,(\bar{u}, 0)) \rightarrow\left(U \times M,\left(u^{*}, 0\right)\right), \alpha_{1}:(U, \bar{u}) \rightarrow$ $\left(U, u^{*}\right)$ such that $u^{*} \in N, \alpha\left(f^{-1}(0)\right)=g^{-1}(0), \alpha(U \times\{0\})=U \times\{0\}$ and $\alpha_{1} \circ \pi_{f}$ $=\pi_{g} \circ \alpha$ at $(\bar{u}, 0)$.

THEOREM 3. Suppose that $(p, p+t-s)$ is a nice dimension when $p+t-s>1$. Then, there exists an open and dense subset $\theta$ in $C^{\infty}\left(U \times M, U \times\{0\} ; R^{t},\{0\}\right)$ such that for any $f$ in $\mathcal{\theta}^{*}$, any $(\bar{u}, 0) \in f^{-1}(0)$, one has

(a) the germ of $f \circ(\psi \times \varphi)^{-1}$ at $(0,0)$ is a relative $V$-universal deformation on $p$ parameters for any product chart $\psi \times \varphi$ on $U \times M$ with $\psi \times \varphi(\bar{u}, 0)=(0,0)$,

(b) the bifurcation map $\pi_{f}: f^{-1}(0) \rightarrow U$ is smoothly stable at $(\bar{u}, 0)$ under small perturbations on $f$.

THEOREM 4. There exists an open and dense subset $\theta^{*}$ in $C^{\infty}(U \times M, U \times$ $\left.\{0\} ; R^{t},\{0\}\right)$ such that for any $f$ in $\theta^{*}$, the bifurcation map $\pi_{f}: f^{-1}(0) \rightarrow U$ is topologically stable at any point $(\bar{u}, 0)$ in $U \times\{0\}$ under smooth perturbations on $f$.

Clearly, one can define the notion about stability of bifurcation map $\pi_{f}$ : $f^{-1}(0) \rightarrow U, f \in C^{\infty}\left(U \times M, R^{t}\right)$ under small perturbations of $f$ in $C^{\infty}\left(U \times M, R^{t}\right)$. It is not difficult to see that for maps $f$ we found in Theorem 1 or Theorem 2, by assertion (b) in each theorem, that $\pi_{f}: f^{-1}(0) \rightarrow U$ is indeed smoothly stable or topologically stable under all small perturbations in each theorem.

3. $V$-universal deformations and relative $V$-universal deformations of map germs. The theory of $V$-universal deformations of map germs has been given in [3]. The relative version of this theory can be obtained by the same ideas without too much difficulty. For the sake of completeness, we formulate both theories and outline only the proofs of this theory in relative version when it seems necessary.

Set $\left(\mathcal{E}_{x}\right)^{t}=\left\{\left(\eta_{1}, \ldots, \eta_{t}\right) \mid \eta_{1}, \ldots, \eta_{t} \in \mathcal{E}_{x}\right\}$ and $\left(m_{x}\right)^{t}=\left\{\left(\eta_{1}, \ldots, \eta_{t}\right) \mid \eta_{1}, \ldots, \eta_{t}\right.$ $\left.\in m_{x}\right\}$, where $\varepsilon_{x}, m_{x}$ are defined in $\$ 2$. Clearly $\left(\varepsilon_{x}\right)^{t},\left(m_{x}\right)^{t}$ are $\varepsilon_{x}$-modules with $\left(m_{x}\right)^{t} \subset\left(\mathcal{E}_{x}\right)^{t}$. Given sets $A \subset \mathcal{E}_{x}, B \subset\left(\mathcal{E}_{x}\right)^{t}$, let $A \cdot B$ denote the set of finite sums of elements in the form $a \cdot b$ with $a \in A$ and $b \in B$. Thus, for $f_{0} \in\left(m_{x}\right)^{t}$ with $x=\left(x_{1}, \ldots, x_{s}\right), \varepsilon_{x}\left\{\partial f_{0} / \partial x_{1}, \ldots, \partial f_{0} / \partial x_{s}\right\}, m_{x}\left\{\partial f_{0} / \partial x_{1}, \ldots, \partial f_{0} / \partial x_{s}\right\}, f_{0}^{*} m_{y}$. $\left(\varepsilon_{x}\right)^{t}$ are $\varepsilon_{x}$-modules. Here $\partial f_{0} / \partial x_{i} \in\left(\mathcal{E}_{x}\right)^{t}$ stands for the partial derivative with respect to $x_{i}$.

Definition 3. Given a smooth map germ $f_{0}:\left(R^{s}, 0\right) \rightarrow\left(R^{t}, 0\right)$ (i.e. $\left.f_{0} \in m_{x}^{t}\right)$, set

$$
T_{V} f_{0}=\varepsilon_{x}\left\{\partial f_{0} / \partial x_{1}, \ldots, \partial f_{0} / \partial x_{s}\right\}+f_{0}^{*} m_{y} \cdot\left(\varepsilon_{x}\right)^{t},
$$


and

$$
\tilde{T}_{V} f_{0}=m_{x}\left\{\partial f_{0} / \partial x_{1}, \ldots, \partial f_{0} / \partial x_{s}\right\}+f_{0}^{*} m_{y} \cdot\left(\varepsilon_{x}\right)^{t} .
$$

$T_{V} f_{0}, \quad \tilde{T}_{V} f_{0}$ are called the $V$-tangent space and relative $V$-tangent space of $f_{0}$, respectively. Obviously, $T_{V} f_{0}, \tilde{T}_{V} f_{0}$ are real vector spaces and $\tilde{T}_{V} f_{0} \subset\left(m_{x}\right)^{t}$. Thus, the quotients $\left(\varepsilon_{x}\right)^{t} / T_{V} f_{0},\left(m_{x}\right)^{t} / \tilde{T}_{V} f_{0}$ are real vector spaces and their dimensions are called the $V$-codimension and the relative $V$-codimension of $f_{0}$ respectively. These codimensions are invariant under $V$-equivalences.

The notion of $V$-universality of map germs can be characterized by transversality conditions, and the existence criterion follows from it easily.

Proposition 1. A deformation (relative deformation) $f$ on p parameters of a smooth map germ $f_{0}:\left(R^{s}, 0\right) \rightarrow\left(R^{t}, 0\right)$ is $V$-universal (relatively $V$-universal) iff $T_{V} f_{0}+$ $R\left\{\dot{f}_{1}, \ldots, \dot{f}_{p}\right\}=\left(\mathcal{E}_{x}\right)^{t}\left(\tilde{T}_{V} f_{0}+R\left\{\dot{f}_{1}, \ldots, \dot{f}_{p}\right\}=\left(m_{x}\right)^{t}\right)$. Here, $\dot{f}_{i}(x)=$ $\left(\partial f / \partial u_{i}\right)(0, x), i=1, \ldots, p$.

COROllaRY 1. Let $f_{0}:\left(R^{s}, 0\right) \rightarrow\left(R^{t}, 0\right)$ be a smooth map germ. Then, there exists $a V$-universal (relative $V$-universal) deformation $f$ on $p$ parameters of $f_{0}$ iff the $V$-codimension (relative $V$-codimension) is less than or equal to $p$.

Applying essentially the same elementary arguments as in Martinet [3], one has the uniqueness results on $V$-universal or relative $V$-universal deformations of map germs.

Proposition 2. Suppose that $f, g:\left(R^{p} \times R^{s}, 0\right) \rightarrow\left(R^{t}, 0\right)$ are two $V$-universal (relative $V$-universal) deformations of a smooth map germ $f_{0}:\left(R^{p}, 0\right) \rightarrow\left(R^{t}, 0\right)$. Then, $f$ is $V$-equivalent (relative $V$-equivalent) to $g$.

Corollary 1 and Proposition 2 imply that the study of $V$-universal deformations or relative $V$-universal deformations on $p$ parameters reduce to the studies of maps with $V$-codimensions or relative $V$-codimensions $<p$. Notice that it has been proved in Mather [4, IV, Theorem 2.5] that for $f_{0}:\left(R^{s}, 0\right) \rightarrow\left(R^{t}, 0\right)$ with rank of $f_{0}<t$, the $V$-codimension of $f_{0}$ plus $s$ is equal to the relative $V$-codimension of $f_{0}$ plus $t$. Let $f_{0}, g_{0}:\left(R^{s}, 0\right) \rightarrow\left(R^{t}, 0\right)$ be two map germs. $f_{0}$ is said to be $V$-equivalent to $g_{0}$ iff there exists a diffeomorphism germ $\varphi:\left(R^{s}, 0\right) \rightarrow\left(R^{s}, 0\right)$ such that $\varphi^{*}\left(g_{0}^{*} m_{y}\right)=f_{0}^{*} m_{y}$.

EXAmple 1. Consider $f_{0}:\left(R^{2}, 0\right) \rightarrow(R, 0)$ with $d f_{0}(0)=0$ and $p=4$. Using essentially the same arguments as in [6], one gets that if the $V$-codimension or the relative $V$-codimension of $f_{0}<4$, then $f_{0}$ is $V$-equivalent to one of the germs in the following diagram.

\begin{tabular}{|c|c|c|}
\hline germ & $V$-codimension & relative $V$-codimension \\
\hline$x^{2} \pm y^{2}$ & 1 & 2 \\
\hline$x^{2} \pm y^{3}$ & 2 & 3 \\
\hline$x^{2} \pm y^{4}$ & 3 & 4 \\
\hline$x^{2} \pm y^{5}$ & 4 & 5 \\
\hline$x^{3} \pm x y^{3}$ & 4 & 5 \\
\hline
\end{tabular}


The $V$-universal deformation and the relative $V$-universal deformation of $x^{2} \pm y^{k}$ $(k>2)$ are given by $x^{2} \pm y^{k}+u_{0}+u_{1} y+\cdots+u_{k-2} y^{k-2}$ and $x^{2} \pm y^{k}+u_{0} x$ $+u_{1} y+\cdots+u_{k-1} y^{k-1}$ respectively. The $V$-universal deformation and the relative $V$-universal deformation of $x^{2} \pm x y^{3}$ are given by $x^{2} \pm x y^{3}+u_{0}+u_{1} x+u_{2} y$ $+u_{3} \cdot\left(x^{2}+y^{2}\right)$ and $x^{2} \pm x y^{3}+u_{1} x+u_{2} y+u_{3} x^{2}+u_{4} x y+u_{5} y^{2}$ respectively.

For the rest of this section, we sketch only the sufficiency of the transversality condition stated in Proposition 1. The necessity of that condition is not hard to prove (cf. [3]).

Let $g:\left(R^{q} \times R^{s}, 0\right) \rightarrow\left(R^{t}, 0\right)$ be any given relative deformation of $f_{0}$. We want to show that there exists a map germ $k:\left(R^{q}, 0\right) \rightarrow\left(R^{p}, 0\right)$ such that $g$ is relatively $V$-isomorphic to $k^{*} f$. Consider a relative deformation $\omega=f \cup g:\left(R^{q} \times R^{p} \times\right.$ $\left.R^{s}, 0\right) \rightarrow\left(R^{t}, 0\right)$ defined by $f \cup g(v, u, x)=f(u, x)+g(v, x)-f_{0}(x)$. Clearly, it suffices to find a submersion $h:\left(R^{q} \times R^{p}, 0\right) \rightarrow\left(R^{p}, 0\right)$ such that $f \cup g$ is relatively $V$-isomorphic to $h^{*} f$. By induction on $q$, it is not difficult to see that one needs only to establish such a submersion $h$ when $q=1$. For this purpose, it suffices to find a smooth vector field germ $X$ in the form

$$
\frac{\partial}{\partial v}+\sum_{i=1}^{p} \xi_{i}(v, u) \frac{\partial}{\partial u_{i}}+\sum_{j=1}^{s} X_{j}(v, u, x) \frac{\partial}{\partial x_{j}}
$$

such that $X_{j}(v, u, 0)=0, D(f \cup g) X \in(f \cup g)^{*} m_{y} \cdot\left(\varepsilon_{v, u, x}\right)^{t}$. The desired submersion $h$ can be obtained from $X$ as $(0, h(v, u))=\varnothing_{-v}(v, u)$ where $\varnothing_{t}(v, u)$ denotes the solution curve of $\partial / \partial v+\sum_{i=1}^{p} \xi_{i}(v, u) \partial / \partial u_{i}$ with $\varnothing_{0}(v, u)=(v, u)$. Set $m_{v, u, x}^{*}=$ $\left\{\omega^{+}:\left(R \times R^{p} \times R^{s}, 0\right) \rightarrow R^{t}\right.$ such that $\left.\omega^{+}(v, u, 0)=0\right\}$. Obviously, $m_{v, u, x}^{*}$ is an ideal in the local ring $\mathcal{E}_{v, u, x}$. Notice that $D \omega(X) \in \omega^{*} m_{y}\left(\mathcal{E}_{v, u, x}\right)^{t}$ means

$$
\frac{\partial \omega}{\partial v}+\sum_{i=1}^{p} \xi_{i}(u, v) \frac{\partial \omega}{\partial u_{i}}+\sum_{j=1}^{s} X_{j}(v, u, x) \frac{\partial \omega}{\partial x_{j}} \in \omega^{*} m_{j}\left(\mathcal{E}_{v, u, x}\right)^{t}
$$

and $\partial(f \cup g) / \partial v$ belongs to $m_{v, u, x}^{*}$. Thus, one can get this vector field $X$ if $\omega=f \cup g$ satisfies condition (A):

$$
\begin{aligned}
\left(m_{v, u, x}^{*}\right)^{t}= & m_{v, u, x}^{*}\left\{\partial \omega / \partial x_{1}, \ldots, \partial \omega / \partial x_{s}\right\} \\
& +\omega^{*} m_{y} \cdot\left(\varepsilon_{v, u, x}\right)^{t}+\varepsilon_{v, u}\left\{\partial \omega / \partial u_{1}, \ldots, \partial \omega / \partial u_{p}\right\} .
\end{aligned}
$$

Therefore, it remains to show that the transversality condition on $f$ implies this condition (A) on $f \cup g$. Now, we need a result of Malgrange. Let $\varphi:\left(R^{a}, 0\right) \rightarrow$ $\left(R^{b}, 0\right)$ with $\varphi(\xi)=\eta$ be a smooth map germ and $M$ a $\mathcal{E}_{\xi}$-module generated by $a_{1}, \ldots, a_{n}$ in $M$. The Malgrange preparation theorem says that the following two conditions (1), (2) are equivalent.

(1) $M$ is generated by $a_{1}, \ldots, a_{n}$ as an $\varepsilon_{\eta}$-module via the homomorphism $\varphi^{*}$ : $\varepsilon_{\eta} \rightarrow \varepsilon_{\xi}$ defined by $\varphi^{*}(\zeta)=\zeta \circ \varphi$.

(2) The quotient space $M / \varphi^{*} m_{y} M$ is spanned by $\bar{a}_{1}, \ldots, \bar{a}_{n}$ as a real vector space. Here $\bar{a}_{i}$ denotes the image of $a_{i}$ under the natural projection $M \rightarrow M / \varphi^{*} m_{y} M$.

Take $\varphi$ as the natural projection $\pi:\left(R \times R^{p} \times R^{s}, 0\right) \rightarrow\left(R \times R^{p}, 0\right)$ given by $\pi(v, u, x)=(v, u)$ and $M$ the quotient $\varepsilon_{v, u, x}$-module

$$
\left(m_{v, u, x}^{*}\right)^{t} / m_{v, u, x}^{*}\left\{\partial \omega / \partial x_{1}, \ldots, \partial \omega / \partial x_{s}\right\}+\omega^{*} m_{y} \cdot\left(\mathcal{E}_{v, u, x}\right)^{t}
$$


Write $\left[\omega^{+}\right]$for the image of $\omega^{+}$under the quotient map from $\left(m_{v, u, x}^{*}\right)^{t}$ onto $M$. Write $[\eta]$ for the image of $\eta$ under the quotient map from $\left(m_{x}\right)^{t}$ onto $\left(m_{x}\right)^{t} / \tilde{T}_{V} f_{0}$. It is not difficult to see that there is $R$-isomorphism $I: M / \pi^{*} m_{y} M \simeq\left(m_{x}\right)^{t} / \tilde{T}_{V} f_{0}$ such that $\overline{I\left[\omega^{+}(v, u, x)\right]}=\left[\omega^{+}(0,0, x)\right]$. The transversality condition on $f$ says that $\left[\partial f_{0} / \partial x_{1}\right], \ldots,\left[\partial f_{0} / \partial x_{s}\right] \operatorname{span}\left(m_{x}\right)^{t} / \tilde{T}_{V} f_{0}$ over $R$. Hence, $\overline{\left[\partial \omega / \partial x_{1}\right]}, \ldots, \overline{\left[\partial \omega / \partial x_{s}\right]}$ span $M / \varphi^{*} m_{y} M$ over $R$. By the Malgrange theorem, one gets that $\left[\partial \omega / \partial x_{1}\right], \ldots,\left[\partial \omega / \partial x_{s}\right]$ generate $M$ as an $\mathcal{E}_{v, u}$-module, which is equivalent to saying that condition (A) holds for $\omega(=f \cup g)$. This completes the outline of the proof of Proposition 1.

4. A transversality theorem and proofs of Theorems 1 and 2. Let $J^{k}(s, t)$ be the set of all polynomials $\xi: R^{s} \rightarrow R^{t}$ of degree $<k$ and $\xi(0)=0$. For $\eta \in m_{x}, j_{0}^{k} \eta$ denotes the $k$ th order Taylor polynomial of $\eta$ at $0 . J^{k}(s, t)$ can be regarded naturally as $R^{l}$ for some positive integer $l$. Denote by $J^{k}\left(M, R^{t}\right)$ the $k$-jet space of smooth mappings from $M$ into $R^{t}$ and $j^{k} g(x)$ the $k$-jet of $g$ at $x$ in $M$ for any $g$ in $C^{\infty}\left(M, R^{t}\right)$. In our case, $M$ is an open set in $R^{s}, J^{k}\left(M, R^{t}\right)=M \times R^{t} \times J^{k}(s, t)$ and $j^{k} g(x)=\left(x, g(x), j_{0}^{k} g(x)\right)$ with $g(x+\xi)=g(x)+j_{0}^{k} g(x)(\xi)+o\left(|\xi|^{k}\right)$. Given any $f \in C^{\infty}\left(U \times M, R^{t}\right)$, set $j_{2}^{k} f(u, x)=j^{k} f_{u}(x)$, where the map $f_{u}: M \rightarrow R^{t}$ is defined by $f_{u}(x)=f(u, x)$. Clearly, the $\operatorname{map} j_{2}^{k} f: U \times M \rightarrow J^{k}\left(M, R^{t}\right)$ is smooth. Now, we state a parametrized version of Thom's transversality theorem, which can be found in [7].

Proposition 3. Given any closed smooth stratified set $C$ in $J^{k}\left(M, R^{t}\right)$, let $\mathcal{\theta}=\left\{f \in C^{\infty}\left(U \times M, R^{t}\right) \mid j_{2}^{k} f\right.$ is transversal to all the strata of $\left.C\right\}$. Then, the set $\theta$ is open and dense in $C^{\infty}\left(U \times M, R^{t}\right)$.

By a stratified set, we mean a set together with a Whitney stratification (without frontier condition) on it. For definition of Whitney stratifications, see [5], [2]. This proposition is needed in the proofs of Theorems 1 and 2.

Next, we present the proof of Theorem 1 stated in \$2. The notion about a contact group $\mathcal{K}^{k}$ acting on $J^{k}(n, p)$, which is introduced by Mather in [4, IV], is needed here. The following facts about $\mathcal{K}^{k}$ are established in [4, V, VI], [5].

(1) The $\mathcal{K}^{k}$-orbit through the $k$-jet $j_{0}^{k} g$ of $g:\left(R^{n}, 0\right) \rightarrow\left(R^{p}, 0\right)$ consists of the $k$-jets of those germs $h:\left(R^{n}, 0\right) \rightarrow\left(R^{p}, 0\right)$ such that $h$ is $V$-equivalent to $g$.

(2) The $\mathcal{K}^{k}$-orbit through $j_{0}^{k} g$ with $g:\left(R^{n}, 0\right) \rightarrow\left(R^{p}, 0\right)$ is a semialgebraic submanifold with tangent space $m_{x}\left\{\partial g / \partial x_{1}, \ldots, \partial g / \partial x_{n}\right\}+g^{*} m_{y}\left(\mathcal{E}_{x}\right)^{p}+$ $\left(m_{x}^{k+1}\right)^{p} /\left(m_{x}^{k+1}\right)^{p}$ in $J^{k}(n, p)$, where $J^{k}(n, p)$ is identified as $\left(m_{x}\right)^{p} /\left(m_{x}^{k+1}\right)^{p}$.

(3) There exists a unique smallest closed algebraic $\mathscr{K}^{k}$-invariant subset $\Pi^{k}(n, p)$ with the property that only finitely many $\mathcal{K}^{k}$-orbits lie outside $\Pi^{k}(n, p)$.

(4) Let $\sigma^{k}(n, p)$ denote the codimension of $\Pi^{k}(n, p)$ in $J^{k}(n, p)$. Let $\sigma(n, p)=$ $\inf _{k} \sigma^{k}(n, p) .(n, p)$ is a nice dimension iff $\sigma(n, p)>n, \sigma(n, p)=\sigma^{k}(n, p)$ for large $k$.

(5) If $j_{0}^{k} f=j_{0}^{k} g \notin \Pi^{k}(n, p)$, then $f$ is $V$-equivalent to $g$ at 0 .

Fix a large $k$ so that $\sigma(s, t)=\sigma^{k}(s, t)$ and $k>p+1$. Let $\Pi^{+}=M \times\{0\} \times$ $\Pi^{k}(s, t)$. Thus, the codimension cod $\Pi^{+}$of $\Pi^{+}$in $J^{k}\left(M, R^{t}\right)$ equals $t+\sigma^{k}(s, t)$ or 
$t+\sigma(s, t)$. Since $(p+s-t, p)$ is a nice dimension by hypothesis, $\sigma(p+s-t, p)$ $>p+s-t$. From the formula about $\sigma($ see $[4, \mathrm{~V}])$, one gets that $\operatorname{cod} \Pi^{+}>p+s$. Denote by $K_{1}, \ldots, K_{a}$ the finite set of $\mathcal{K}^{k}$-orbits outside $\Pi^{k}(s, t)$. By basic theorems on stratifications of semialgebraic sets cf. [5], one can get a Whitney stratification $\delta$ on $J^{k}(s, t)$ so that $K_{1}, \ldots, K_{a}$ are strata and $\Pi^{k}(s, t)$ is a finite union of strata. Let $C=M \times\{0\} \times J^{k}(s, t)$ be the stratified set with the stratification $\mathcal{S}^{+}=\{M \times\{0\} \times S \mid S \in \mathcal{S}\}$. Proposition 3 implies that the set $\mathcal{\theta}=\{f$ $\in C^{\infty}\left(U \times M, R^{t}\right) \mid j_{2}^{k} f$ is transversal to all strata in $\left.\delta^{+}\right\}$is open and dense. We claim that this set $\mathcal{\theta}$ has desired properties. For the assertion (a) it suffices to observe that $M \times\{0\} \times J^{k}(s, t)$ is a submanifold of codimension $t$. The justification of assertion (b) requires the Nakayama Lemma in the following form: Let $M, N$ be two submodules in some $\mathcal{E}_{x}$-module with $M$ finitely generated over $\mathcal{E}_{x}$, then $M \subset N+m_{x} M$ implies that $M \subset N$. Let $(u, x) \in f^{-1}(0)$ and set $\tilde{f}=f \circ$ $(\psi \times \varphi)^{-1}$. From the established fact $\operatorname{cod} \Pi^{+}>p+s$, we get $j_{2}^{k} \tilde{f}(0,0) \in K_{i}^{+}$for some $i . \quad j_{2}^{k} \tilde{f}$ is transversal to $K_{i}^{+}$at $(u, x)$ means that

$$
\begin{aligned}
R\left\{\left(0, \dot{\tilde{f}}_{1}\right), \ldots,\right. & \left.(0, \dot{\tilde{f}}),\left(e_{1}, \partial \tilde{f}_{0} / \partial x_{1}\right), \ldots,\left(e_{s}, \partial \tilde{f}_{0} / \partial x_{s}\right)\right\} \\
& +\{0\} \times\left[m_{x}\left\{\partial \tilde{f}_{0} / \partial x_{1}, \ldots, \partial \tilde{f}_{0} / \partial x_{s}\right\}+\tilde{f}_{0}^{*} m_{y}\left(\varepsilon_{x}\right)^{t}\right] \\
& +\{0\} \times\left(m_{x}^{k+1}\right)^{t}=R^{s} \times\left(\varepsilon_{x}\right)^{t} .
\end{aligned}
$$

Here, $e_{i}$ denotes the $i$ th unit vector in $R^{s}$. This transversality condition is equivalent to the condition

$$
R\left\{\tilde{f}_{1}, \ldots, \tilde{f}_{p}\right\}+\mathcal{E}_{x}\left\{\partial \tilde{f}_{0} / \partial x_{1}, \ldots, \partial \tilde{f}_{0} / \partial x_{s}\right\}+\tilde{f}_{0}^{*} m_{y}\left(\mathcal{E}_{x}\right)^{t}+\left(m_{x}^{k+1}\right)^{t}=\left(\mathcal{E}_{x}\right)^{t}
$$

Since $k>p+1$,

$$
\begin{aligned}
\mathcal{E}_{x}\left\{\partial \tilde{f}_{0} / \partial x_{1}, \ldots, \partial \tilde{f}_{0} / \partial x_{s}\right\}+\tilde{f}_{0}^{*} m_{y}\left(\mathcal{E}_{x}\right)^{t}+\left(m_{x}^{j}\right)^{t} & =\mathcal{E}_{x}\left\{\partial \tilde{f}_{0} / \partial x_{1}, \ldots, \partial \tilde{f}_{0} / \partial x_{s}\right\} \\
& +\tilde{f}_{0}^{*} m_{y}\left(\mathcal{E}_{x}\right)^{t}+\left(m_{x}^{j+1}\right)^{t} \text { for some } 1<j<k .
\end{aligned}
$$

Using the Nakayama lemma with $N=\mathcal{E}_{x}\left\{\partial \tilde{f}_{0} / \partial x_{1}, \ldots, \partial \tilde{f}_{0} / \partial x_{s}\right\}+\tilde{f}_{0}^{*} m_{y}\left(\mathcal{E}_{x}\right)^{t}$, $M=\left(m_{x}^{j}\right)^{t}$, we obtain $R\left\{\dot{\tilde{f}}_{1}, \ldots, \dot{\tilde{f}}_{p}\right\}+\tilde{f}_{0}^{*} m_{y}\left(\mathcal{E}_{x}\right)^{t}=\left(\mathcal{E}_{x}\right)^{t}$. By Proposition 1, we know that $\tilde{f}$ defines a $V$-universal deformation of $\tilde{f}_{0}$ at $(0,0)$. Consequently, we finish the proof of Theorem 1 .

At the end of this section we give the proof of Theorem 2 and a remark. Here we need the stratification given in Looijenga [2], which is constructed for the purpose of establishing genericity of topologically stable maps. Recall that $J^{k}(m, n)$ is decomposed into a disjoint union of $S_{j}(m, n), j=0,1,2, \ldots$, and $W^{k}(m, n)$ with the following properties [2].

(1) $W^{k}(m, n)$ is an algebraic variety and the codimension $W^{k}(m, n)$ in $J^{k}(m, n)$ tends to $\infty$ when $k$ tends to $\infty$.

(2) $S_{j}, j=0,1, \ldots, W^{k}$, are invariant under $\mathcal{K}^{k}$ action on $J^{k}(m, n)$ and $\left\{S_{j}\right\}$ defines a Whitney stratification (without frontier condition).

(3) Let id be the identity map on $R^{l}$. Then $j_{0}^{k} \eta \in S_{j}(m, n)$ iff $j_{0}^{k}(\mathrm{id} \times \eta) \in$ $S_{j}(m+l, n+l)$. 
(4) A smooth map-germ $\eta:\left(R^{m}, 0\right) \rightarrow\left(R^{n}, 0\right)$ is topologically stable if for some representative $f$ of $\eta, j_{0}^{k} f(0) \in S_{j}(m, n)$ and $j_{0}^{k} f$ is transversal to $S_{j}(m, n)$ in $J^{k}(m, n)$ at 0 .

Now, choose $k$ large so that $\operatorname{cod} W^{k}(s, t)>p+s-t$. Set $\theta=\{f \in$ $C^{\infty}\left(U \times M, R^{t}\right) \mid j_{2}^{k} f(U \times M) \cap W^{+}=\varnothing, j_{2}^{k} f$ is transversal to $S_{j}^{+}, j=$ $0,1, \ldots\}$, where $W^{+}=M \times\{0\} \times W^{k}(s, t)$ and $S_{j}^{+}=M \times\{0\} \times S_{j}(s, t)$. Since $\operatorname{cod} W^{+}>p+s$, by (a slight variant of) Proposition 3, we know that $\theta$ is open and dense. We claim that this set $\theta$ has desired properties. Take a map $f$ in $\theta$ and fix any $(\bar{u}, \bar{x})$ in $f^{-1}(0) . j_{2}^{k} f$ is transversal to $S_{j}^{+}$at $(\bar{u}, \bar{x})$ for some $j$. For assertion (a) it suffices to observe that $M \times\{0\} \times J^{k}(s, t)$ is a submanifold of codimension $t$ and $M \times\{0\} \times J^{k}(s, t)$ contains $S_{j}^{+}$. To prove assertion (b), we begin by establishing a lemma about $S_{j}$. Denote by $G$ the open set $\left\{j_{0}^{k}(h, g) \in\right.$ $J^{k}(p+s, p+t) \mid h:\left(R^{p+s}, 0\right) \rightarrow\left(R^{p}, 0\right), g:\left(R^{p+s}, 0\right) \rightarrow\left(R^{t}, 0\right)$, and $(\partial h / \partial u)(0)$ is nonsingular $\}$ in $J^{k}(p+s, p+t)$, here $(u, x)$ stands for the natural coordinates on $R^{p} \times R^{s}$. For $j^{k}(h, g)$ in $G$, the nonsingularity of the $p \times p$ matrix $(\partial h / \partial u)(0)$ implies that we can solve uniquely the equation $h(u, x)=0$ close to the origin by $u=u^{*}(x)$. Define a smooth (algebraic) map $\pi: G \rightarrow J^{k}(p, t)$ by $\pi\left(j_{0}^{k}(h, g)\right)=j_{0}^{k} g^{*}$ with $g^{*}(x)=g\left(u^{*}(x), x\right)$.

LEMMA 1. $\pi: G \rightarrow J^{k}(p, t)$ is a submersion and

$$
\pi^{-1}\left(S_{j}(p, t)\right)=G \cap S_{j}(p+s, p+t) .
$$

Define $\rho: J^{k}(p, t) \rightarrow G$ by $\rho\left(j_{0}^{k} g\right)=j_{0}^{k}(\mathrm{id} \times g)$ with $\operatorname{id}(u)=u$ on $R^{p}$. Clearly, $\pi \circ \rho$ is an identity map on $J^{k}(p, t)$. Thus, $\pi$ is a submersion. Take any $j^{k}(h, g)$ in $G$ and write $\varphi$ for the local diffeomorphism $\left(\begin{array}{l}u \\ x\end{array}\right) \rightarrow\left(\begin{array}{l}h \\ x\end{array}\right)$. Hence, $u$ can also be regarded as map $u(h, x)$ of the variables $(u, x)$ and $u(0, x)$ is equal to $u^{*}(x)$. The map germ $\left(\begin{array}{l}u \\ x\end{array}\right) \rightarrow\left(\begin{array}{l}h \\ g\end{array}\right)$ is $V$-equivalent to the map germ $\left(\begin{array}{l}h \\ x\end{array}\right) \rightarrow(g(u(h, x)), x)$ via the local diffeomorphism $\varphi$. The map germ $\left(\begin{array}{l}h \\ x\end{array}\right) \rightarrow\left(\begin{array}{c}g(u(h, x)), x \\ h\end{array}\right)$ is $V$-equivalent to $\left(\begin{array}{l}h \\ x\end{array}\right) \rightarrow$ $\left(\begin{array}{c}h(u, 0, x)), x \\ )\end{array}\right.$ or $\left(\begin{array}{c}h \\ x\end{array}\right) \rightarrow\left(\begin{array}{c}h \\ g^{*}(x)\end{array}\right)$. Therefore, we get $j_{0}^{k} g^{*} \in S_{j}(p, t)$ iff $j_{0}^{k}(h, g) \in$ $S_{j}(p+s, p+t)$. Consequently, $\pi^{-1}\left(S_{j}(p, t)\right)=S_{j}(p+s, p+t) \cap G$ which finishes the proof of Lemma 1.

Let $\pi^{+}: U \times M \times R^{p} \times R^{t} \times G \rightarrow M \times R^{t} \times J^{k}(s, t)$ be the submersion given by $\pi^{+}(u, x, a, b, z)=(x, b, \pi(z))$. Write $F$ for the map $F(u, x)=(u, f(u, x))$ from $U \times M$ into $U \times R^{t}$. Since $\pi^{+} \circ j^{k} F=j_{2}^{k} f$ on $U \times M$, by Lemma 1 , we obtain that $j_{2}^{k} f$ is transversal to $S_{j}^{+}$in $J^{k}\left(M, R^{t}\right)$ at $(\bar{u}, \bar{x})$ iff $j^{k} F$ is transversal to $U \times M \times R^{p} \times\{0\} \times S_{j}$ in $J^{k}\left(U \times M, R^{p} \times R^{t}\right)$ at $(\bar{u}, \bar{x})$. The fact that $j^{k} F$ is transversal to $U \times M \times R^{p} \times\{0\} \times S_{j}$ at $(\bar{u}, \bar{x})$ implies $j_{0}^{k} F: f^{-1}(0) \rightarrow$ $J^{k}(p+s, p+t)$ is transversal to $S_{j}(p+s, p+t)$ at $(\bar{u}, \bar{x})$. Without loss of generality, we may assume that $(u, x)=\left(u_{1}, u_{2}, x_{1}, x_{2}\right)$ and $\left(\partial f / \partial\left(u_{2}, x_{2}\right)\right)(\bar{u}, \bar{x})$ is nonsingular. Denote by $G^{*}$ the open set $\left\{j_{0}^{k}(h, g) \in J^{k}(p+s, p+t) \mid\left(\partial g / \partial\left(u_{2}, x_{2}\right)\right)(0)\right.$ is nonsingular $\}$ in $J^{k}(p+s, p+t)$. Define $\pi^{*}: G^{*} \rightarrow J^{k}(p+s-t, p)$ by $\pi^{*}\left(j_{0}^{k}(h, g)\right)=j_{0}^{k} g^{*}$ with $g^{*}\left(u_{1}, x_{1}\right)=g\left(u_{1}, u_{2}^{*}\left(u_{1}, x_{1}\right), x_{1}, x_{2}^{*}\left(u_{1}, x_{1}\right)\right)$. Here, $u_{2}=$ $u_{2}^{*}\left(u_{1}, x_{1}\right), x_{2}=x_{2}^{*}\left(u_{1}, x_{1}\right)$ solve the equation $g\left(u_{1}, u_{2}, x_{1}, x_{2}\right)=0$ near via implicit function theorem. By Lemma 1 (up to a permutation of variables), we know that $\pi^{*}$ is a submersion and $\left(\pi^{*}\right)^{-1}\left(S_{j}\right)=S_{j} \cap G^{*}$. Therefore, 


$$
\pi^{*} \circ j_{0}^{k} F: f^{-1}(0) \rightarrow J^{k}(p+s, p+t)
$$

is transversal to $S_{j}(p+s, p+t)$ at $(\bar{u}, \bar{x})$. Since $\left(\partial f / \partial\left(u_{2}, x_{2}\right)\right)(\bar{u}, \bar{x})$ is nonsingular, the restriction of the map $\left(u_{1}, x_{1}\right)$ on $f^{-1}(0)$ defines a chart $\psi$ on a neighborhood $N$ of $(\bar{u}, \bar{x})$ in $f^{-1}(0)$. It is not hard to see that $\pi^{*} \circ j_{0}^{k} F=j_{0}^{k}\left(u_{1}, u_{2}^{*}\right) \circ \psi$ on $N$, where $\left(u_{1}, u_{2}^{*}\right): \psi(N) \rightarrow R^{p}$ is given by $\left(u_{1}, u_{2}^{*}\right)\left(u_{1}, x_{1}\right)=\left(u_{1}, u_{2}^{*}\left(u_{1}, x_{1}\right)\right)$. Consequently, $j_{0}^{k}\left(u_{1}, u_{2}^{*}\right)$ is transversal to $S_{j}$ at $(\bar{u}, \bar{x})$ and the bifurcation map $\pi=\left(u_{1}, u_{2}^{*}\right) \circ \psi$ : $f^{-1}(0) \rightarrow U$ is topologically stable at $(\bar{u}, \bar{x})$. Hence, we complete the proof of Theorem 2.

REMARK 1. Most arguments in the proof of Theorem 3 are still valid when one replaces the stratification $\left\{S_{j}\right\}$ by $\left\{K_{i}\right\}$. In particular, one obtains that given a smooth germ $f:\left(R^{p} \times R^{s}, 0\right) \rightarrow\left(R^{t}, 0\right)$ with $d f(0)\left(R^{p} \times R^{s}\right)=R^{t}$, then $f$ is a universal deformation iff the bifurcation map $\pi_{f}: f^{-1}(0) \rightarrow R^{p}$ is smoothly stable.

5. A relative transversality theorem and the proofs of Theorems 3 and 4. Recall that $C^{\infty}\left(U \times M, U \times\{0\} ; R^{t},\{0\}\right)$ denotes the (Baire) space of those smooth maps $f$ from $U \times M$ into $R^{t}$ with $f(U \times\{0\})=\{0\}$, where $U, M$ are open sets in $R^{p}, R^{s}$ respectively, $0 \in M$ and $p+t-s \geqslant 1$. The following relative version of Proposition 3, which is needed in the proofs of Theorems 3 and 4, can be proved by similar methods.

Proposition 4. Let $C$ be a closed tratified set in $M \times R^{t} \times J^{k}(s, t)$ such that for any stratum $S$ in $C, S \cap\{0\} \times R^{t} \times J^{k}(s, t) \neq \varnothing$ implies that $S=\{0\} \times R^{t} \times A$ for some submanifold $A$ in $J^{k}(s, t)$. Put $\theta^{*}=\left\{f \in C^{\infty}(U \times M, U \times\right.$ $\left.\{0\} ; R^{t},\{0\}\right) \mid j_{2}^{k} f: U \times M \rightarrow M \times R^{t} \times J^{k}(s, t)$ is transversal to all strata in $\left.C\right\}$. Then the set $\mathcal{O}^{*}$ is open and dense in $C^{\infty}\left(U \times M, U \times\{0\} ; R^{t},\{0\}\right)$.

Now, we give the proof of Theorem 3 as stated in $\$ 2$. Recall that $\Pi^{k}(s, t)$ stands for the unique smallest closed algebraic $\mathcal{K}^{k}$-invariant subset in $J^{k}(s, t)$ with the property that only finitely many $\mathcal{K}^{k}$-orbits lie outside $\Pi^{k}(s, t), \sigma(s, t)=\inf \sigma^{k}(s, t)$, where $\sigma^{k}(s, t)$ is the codimension of $\Pi^{k}(s, t)$ in $J^{k}(s, t)$. Fix a large $k$ so that $\sigma(s, t)=\sigma^{k}(s, t)$ and $k \geqslant p+1$. Denote by $K_{1}, \ldots, K_{a}$ the finitely many $\mathcal{K}^{k}$ orbits lying outside $\Pi^{k}(s, t)=\Pi(s, t)$. Choose a Whitney stratification $\mathcal{S}$ on $J^{k}(s, t)$ such that $K_{1}, \ldots, K_{a}$ are strata and $\Pi^{k}(s, t)$ is a finite union of strata. Put $S^{*}=\{0\} \times R^{t} \times S$ for any set $S$ in $J^{k}(s, t)$. Clearly, the collection $\left\{S^{*} \mid S \in \delta\right\}$ forms a Whitney stratification of the closed set $C=\{0\} \times R^{t} \times J^{k}(s, t)$. Using the condition that $(p, p+t-s)$ is a nice dimension, we get $\operatorname{cod} \Pi^{*}>p+s$. Thus, the fact that $j_{2}^{k} f$ is transversal to $S^{*}$ for a stratum $S$ in $\Pi^{*}$ simply means that $j_{2}^{k} f(U \times M) \cap S^{*}=\varnothing$. Applying Proposition 4 to the stratified set $C$, we obtain that the set

$$
\begin{aligned}
\theta^{*}=\left\{f \in C^{\infty}\left(U \times M, U \times\{0\} ; R^{t},\{0\}\right) \mid\right. \\
\left.\qquad j_{2}^{k} f \text { is transversal to } K_{i}^{*} \text { for all } i, j_{2}^{k} f(U \times M) \cap \Pi^{*}=\varnothing\right\}
\end{aligned}
$$

is both open and dense in $C^{\infty}\left(U \times M, U \times\{0\} ; R^{t},\{0\}\right)$. We claim that the set $\theta^{*}$ gets all the properties we want. Let $f \in \mathcal{\theta}^{*}, j_{2}^{k} f(u, 0) \in K_{i}^{*}$ for some $i$, and $\tilde{f}=f \circ(\psi \times \varphi)^{-1} \cdot j_{2}^{k} \tilde{f}$ is transversal to $K_{i}^{*}$ at $(0,0)$ means that 
$\{0\} \times R^{t} \times\left[m_{x}\left\{\partial \tilde{f}_{0} / \partial x_{1}, \ldots, \partial \tilde{f}_{0} / \partial x_{s}\right\}+\tilde{f}^{*} m_{y} \cdot\left(\mathcal{E}_{x}\right)^{t}\right]$

$$
\begin{aligned}
& +R\left\{\left(0,0, \partial f / \partial u_{1}\right), \ldots,\left(e_{1},\left(\partial \tilde{f}_{0} / \partial x_{1}\right)(0,0), \partial \tilde{f}_{0} / \partial x_{1}-\left(\partial \tilde{f}_{0} / \partial x_{1}\right)(0,0)\right), \ldots\right\} \\
& +\{0\} \times\{0\} \times\left(m_{x}^{k+1}\right)^{t}=R^{s} \times R^{t} \times\left(m_{x}\right)^{t} .
\end{aligned}
$$

This transversality condition is equivalent to

$$
\begin{aligned}
m_{x}\left\{\partial \tilde{f}_{0} / \partial x_{1}, \ldots, \partial \tilde{f}_{0} / \partial x_{s}\right\}+\tilde{f}^{*} m_{y} \cdot\left(\mathcal{E}_{x}\right)^{t} & \\
& +R\left\{\partial \tilde{f} / \partial u_{1}, \ldots, \partial \tilde{f} / \partial u_{p}\right\}+\left(m_{x}^{k+1}\right)^{t}=\left(m_{x}\right)^{t} .
\end{aligned}
$$

Since $k>p+1$, we know that

$$
\begin{aligned}
& m_{x}\left\{\partial \tilde{f}_{0} / \partial x_{1}, \ldots, \partial \tilde{f}_{0} / \partial x_{s}\right\}+\tilde{f}^{*} m_{y}\left(\mathcal{E}_{x}\right)^{t}+\left(m_{x}^{j}\right)^{t} \\
& \quad=m_{x}\left\{\partial \tilde{f}_{0} / \partial x_{1}, \ldots, \partial \tilde{f}_{0} / \partial x_{s}\right\}+\tilde{f}^{*} m_{y} \cdot\left(\mathcal{E}_{x}\right)^{t}+\left(m_{x}^{j+1}\right)^{t} \text { for some } 1<j<k .
\end{aligned}
$$

Using Nakayama's lemma, we obtain that

$$
m_{x}\left\{\partial \tilde{f}_{0} / \partial x_{1}, \ldots, \partial \tilde{f}_{0} / \partial x_{s}\right\}+\tilde{f}^{*} m_{y} \cdot\left(\mathcal{E}_{x}\right)^{t}+R\left\{\partial \tilde{f} / \partial u_{1}, \ldots, \partial \tilde{f} / \partial u_{p}\right\}=\left(m_{x}\right)^{t}
$$

or

$$
\tilde{T}_{V} \tilde{f}_{0}+R\left\{\dot{\tilde{f}}_{1}, \ldots, \dot{\tilde{f}}_{p}\right\}=\left(m_{x}\right)^{t}
$$

By Proposition 1, $\tilde{f}$ defines a relative $V$-universal deformation of $\tilde{f}_{0}$ at $(0,0)$. Thus, assertion (a) about $f$ in $\theta^{*}$ is justified. Let $N$ be a small neighborhood of $(\bar{u}, 0)$ in $U$. Since $j_{2}^{k} f$ is transversal to $K_{i}^{*}$, for $g$ sufficiently close to $f, j_{2}^{k} g$ is also transversal to $K_{i}^{*}$ at $\left(u^{*}, 0\right)$ for some $u^{*} \in N$. Since $j_{0}^{k} f_{0}$ and $j_{0}^{k} g_{0}$ belong to $K_{i}$, they are in fact $V$-equivalent to each other (from properties (1), (5) about $\mathscr{K}^{k}$ group stated in $\S 4$ ). By uniqueness of relative $V$-universal deformations (Proposition 2), we conclude that there exists diffeomorphism $\alpha: N_{1} \rightarrow N_{2}$ around $(u, 0)$ in the form $\alpha(u, x)=$ $\left(\alpha_{1}(u), \quad \alpha_{2}(u, x)\right)$ with $\alpha_{1}(\bar{u})=u^{*}, \quad \alpha_{2}(u, 0)=0$, and $\alpha_{1}\left(N_{1} \cap f^{-1}(0)\right)=N_{2} \cap$ $g^{-1}(0)$. Thus, $\pi_{g} \circ \alpha=\alpha_{1} \circ \pi_{f}$. Therefore, we establish assertion (b), which says $\pi_{f}$ : $f^{-1}(0) \rightarrow U$ is smoothly stable at $(\bar{u}, 0)$ under small perturbations of $f$. Consequently, the proof of Theorem 3 is completed.

Thirdly, we present the proof of Theorem 4. Again, let $S_{j}, j=0,1, \ldots, W^{k}(s, t)$ be the partition of $J^{k}(s, t)$ given in Looijenga [2]. Fix large $k$ so that $\sigma(s, t)=$ $\sigma^{k}(s, t)$ and $\sigma^{k}(s, t)>p$. Put $W^{*}=\{0\} \times R^{t} \times W^{k}(s, t), S_{j}^{*}=\{0\} \times R^{t} \times S_{j}$, $j=0,1, \ldots$ Clearly that cod $W^{*}>p+s$, and, by Proposition 4, the set $\theta^{*}=\{f$ $\in C^{\infty}\left(U \times M, U \times\{0\} ; R^{t},\{0\}\right) \mid j_{2}^{k} f(U \times M) \cap W^{*}=\varnothing, j_{2}^{k} f$ is transversal to $S_{j}^{*}$, for $\left.j=0,1, \ldots\right\}$ is open and dense. It remains to show that for any $f$ in $\theta^{*}$, $\bar{u} \in U$, the bifurcation map $\pi_{f}: f^{-1}(0) \rightarrow U$ is topologically stable at $(\bar{u}, 0)$ under small perturbations of $f$. Recall the relationship $\pi^{+} \circ j^{k} F=j_{2}^{k} f$ used in the proof of Theorem 2, where $\pi^{+}$is a submersion. Thus, $j_{2}^{k} f$ is transversal to $S_{j}^{*}$ at $(\bar{u}, 0)$ implies that $j^{k} F$ is transversal to $U \times\{0\} \times R^{p} \times R^{t} \times S_{j}(p+s, p+t)$. Let $N$ be a small neighborhood of $(\bar{u}, 0)$. For $g$ sufficiently close to $f$, the map $G$ : 
$U \times M \rightarrow U \times R^{t}$ defined by $G(u, x)=(u, g(u, x))$ is sufficiently close to $F$. Therefore, $G$ is also transversal to $U \times\{0\} \times R^{p} \times R^{t} \times S_{j}(p+s, p+t)$ at some point $\left(u^{*}, 0\right)$ with $u^{*}$ in $N$. Notice that the Whitney stratification $\{U \times$ $\left.(M \backslash\{0\}) \times R^{p} \times R^{t} \times S_{j} \mid j=0,1, \ldots\right\} \cup\left\{U \times\{0\} \times R^{p} \times R^{t} \times S_{j} \mid j=\right.$ $0,1, \ldots\}$ refines the Whitney stratification $\left\{U \times M \times R^{p} \times R^{t} \times S_{j} \mid j=\right.$ $0, ?, \ldots\}$. By the same arguments as in Looijenga [2], we obtain strata-preserving diffeomorphisms $\alpha: N_{1} \rightarrow N_{2}, \beta: N_{3} \rightarrow N_{4}$ on neighborhoods $N_{1}, N_{3}$ of $(\bar{u}, 0)$, $F(\bar{u}, 0)$ in $U \times M, U \times R^{t}$ respectively such that $\beta \circ F=G \circ \alpha$ on $N_{1}$. In particular, $\alpha\left(N_{1} \cap U \times\{0\}\right)=N_{2} \cap U \times\{0\}$. Put $N_{1}^{*}=\left\{u \in U \mid(u, 0) \in N_{1}\right\}$, $N_{2}^{*}=\left\{u \in U \mid(u, 0) \in N_{2}\right\}$. Shrinking $N_{1}, N_{2}$ if necessary, one may assume that $N_{i} \subset N_{i}^{*} \times M$ for $i=1,2$. Let $\alpha_{1}: N_{1}^{*} \rightarrow N_{2}^{*}$ be the diffeomorphism defined by $\alpha(u, 0)=\left(\alpha_{1}(u), 0\right)$. From the facts $\alpha\left(N_{1} \cap U \times\{0\}\right)=N_{2} \cap(U \times\{0\}), \beta \circ F$ $=G \circ \alpha$, and $F(u, 0)=(u, 0), G(u, 0)=(u, 0)$ on $U$, we get $\alpha\left(N_{1} \cap f^{-1}(0)\right)=N_{2}$ $\cap g^{-1}(0)$ and $\alpha_{1} \circ \pi_{f}=\pi_{g} \circ \alpha$ on $N_{1} \cap f^{-1}(0)$. Hence, $\pi_{f}$ is topologically stable at $(\bar{u}, 0)$ under small perturbations on $f$. Consequently, the proof of Theorem 4 is completed.

At the end of this paper, we give examples which show that when the dimensions $p$ of the parameter spaces are large the conclusions of Theorems 1 and 3 may fail.

EXAmple 2. Consider the map $f: R^{8} \times R^{2} \rightarrow R$ defined by $f(u, x, y)=x^{4}+y^{4}$ $+u_{0}+u_{1} x+u_{2} y+u_{3} x^{2}+u_{4} x y+u_{5} y^{2}+u_{6} x^{2} y+u_{7} x y^{2}$. We claim that if $g$ is sufficiently close to $f$, then $g$ is not a $V$-universal deformation at some point $(\bar{u}, \bar{x}, \bar{y}) \in f^{-1}(0)$ close to $(0,0,0)$, i.e., for any product chart $\psi \times \varphi$ on $R^{8} \times R^{2}$ with $\psi \times \varphi(\bar{u}, \bar{x}, \bar{y})=(0,0,0), g \circ(\psi \times \varphi)^{-1}$ is not a $V$-universal deformation at $(0,0)$. Denote by $\mathcal{F}^{(4)} f_{\lambda}$ the $\mathcal{K}^{(4)}$ orbit through $f_{\lambda}(x, y)=x^{4}+y^{4}+\lambda x^{2} y^{2}$. Computations show that if $\mathscr{K}^{(4)} \tilde{g}=\mathscr{K}^{(4)} f_{\lambda}$ for some (small) $\lambda$ then the $V$-codimension of $\tilde{g}$ is equal to 9. Put $W=\cup_{\lambda} \mathscr{K}^{(4)} f_{\lambda}$ and $W^{+}=R^{2} \times\{0\} \times W . W, W^{+}$are manifolds and $j_{2}^{(4)} f$ is transversal to $W^{+}$. If $g$ is sufficiently close to $f$, then $j_{2}^{(4)} g$ is also transversal to $W^{+}$at some point $(\bar{u}, \bar{x}, \bar{y})$ near $(0,0,0)$ with $j_{2}^{(4)} g(\bar{u}, \bar{x}, \bar{y}) \in W^{+}$. Let $\psi \times \varphi$ be any product chart $\psi \times \varphi$ on $R^{8} \times R^{2}$ with $(\psi \times \varphi)(\bar{u}, \bar{x}, \bar{y})=$ $(0,0,0)$, and set $\tilde{g}=g \circ(\psi \times \varphi)^{-1} \cdot j_{2}^{(4)} g(\bar{u}, \bar{x}, \bar{y}) \in W^{+}$implies that $\mathscr{K}^{(4)} \tilde{g}=\mathscr{K}^{(4)} f_{\lambda}$ for some $\lambda$. Therefore, the $V$-codimension of $\tilde{g}$ is equal to 9. By Proposition 1, we conclude that $\tilde{g}$ is not a $V$-universal deformation at $(0,0,0)$.

EXAMPLE 3. Consider the map $f: R^{9} \times R^{2} \rightarrow R$ defined by $x^{4}+y^{4}+u_{1} x+u_{2} y$ $+u_{3} x^{3}+u_{4} x y+u_{5} y^{2}+u_{6} x^{2} y+u_{7} x y^{2}+u_{8} x^{3}+u_{9} y^{3}$. We claim that if $g: R^{9} \times$ $R^{2} \rightarrow R$ with $g\left(R^{9}, 0\right)=0$ is sufficiently close to $f$, then $g$ is not a relative $V$-universal deformation at some point $(\bar{u}, 0,0)$ near $(0,0,0)$. Computations show that if $\mathscr{K}^{(4)} \tilde{g}=\mathscr{K}^{(4)} f_{\lambda}$ for some (small) $\lambda$, then the relative $V$-codimension of $\tilde{\boldsymbol{g}}$ is equal to 10. Put $W^{*}=\{0\} \times R \times W$ with $W=\cup_{\lambda} \mathscr{K}^{(4)} f_{\lambda}$, then $W^{*}$ is a manifold and $j_{2}^{(4)} g$ is transversal to $W^{*}$. When $g$ is sufficiently close to $f$ with $g\left(R^{9}, 0\right)=0, g$ is also transversal to $W^{*}$ at some point $\left(u^{*}, 0,0\right)$ near $(0,0,0)$ with $j_{2}^{(4)} g\left(u^{*}, 0,0\right) \in$ $W^{*}$. Thus, $\mathcal{K}^{(4)} \tilde{g}=\mathcal{K}^{(4)} f_{\lambda}$ for some $\lambda$, where $\tilde{g}=g \circ(\psi \times \varphi)^{-1}$ and $\psi \times \varphi$ can be any product chart on $R^{9} \times R^{2}$ with $\psi \times \varphi\left(u^{*}, 0,0\right)=(0,0,0)$. Thus, the relative $V$-codimension of $\tilde{g}$ is equal to 10 . By Proposition 1 , we get that $g$ cannot be a relative $V$-universal deformation at $\left(u^{*}, 0,0\right)$. 


\section{REFERENCES}

1. G. Debreu, Excess demand functions, J. Math. Econom. 1 (1974), 15-22.

2. E. Looijenga, Proof of the topological stability theorem in topological stability of smooth mappings, Lecture Notes in Math., vol. 552, Springer-Verlag, Berlin and New York, 1976.

3. J. Martinet, Déploiements versels des applications différentiables et classification des applications stables, Singularités d'Applications Différentiables, Lecture Notes in Math., vol. 535, Springer-Verlag, Berlin and New York, 1976.

4. J. N. Mather, Stability of mappings. IV-VI.

IV. Inst. Hautes Etudes Sci. Publ. Math. 37 (1970), 223-248.

V. Advances in Math. 4 (1970), 301-335.

VI. Proc. Liverpool Singularities-Symposium, I (1969-1970), Lecture Notes in Math., vol. 192, Springer, Berlin, 1971, pp. 207-253.

5. , Stratifications and mappings, Dynamical Systems, Academic Press, New York and London, 1973.

6. G. Wassermann, Stability of unfoldings, Lecture Notes in Math., vol. 393, Springer-Verlag, Berlin and New York, 1974.

7. C. Zeeman, The classification of elementary catastrophes of codimension < 5, Structural Stability, the Theory of Catastrophes and Applications in the Sciences, Lecture Notes in Math., vol. 525, SpringerVerlag, Berlin and New York, 1975.

Department of Mathematics, State University of New York at Buffalo, Bupfalo, New York 14214 